\title{
Normal activation of the supplementary motor area in patients with Parkinson's disease undergoing long-term treatment with levodopa
}

\author{
O Rascol, U Sabatini, F Chollet, N Fabre, J M Senard, J L Montastruc, P Celsis, \\ J P Marc-Vergnes, A Rascol
}

Laboratory of Medical and Clinical

Pharmacology, Faculty of Medicine, National Institute of Health and Medical Research, INSERM U317

O Rascol

J M Senard

J L Montastruc

INSERM U230,

U Sabatini

$P$ Celsis

J P Marc-Vergnes

Neurological

Departments,

University Hospital

Center, Toulouse,

France

F Chollet

N Fabre

A Rascol

Department of

Neurological Sciences,

University "La

Sapienza", Rome,

Italy

U Sabatini

Correspondence to:

Professor Olivier Rascol,

Paboratorie de

Pharmacologie Medicale et
Clinique, INSERM U317,

Clinique, INSERM U317,

Faculté de Médecine, 37
allées Jules-Guesde, 31073

Received 1 March 1993

and in revised form

8 September 1993.

8 September 1993. Toulouse, France

allées Jules-Guesde, 31073

\begin{abstract}
Regional cerebral blood flow (rCBF) changes in cortical motor areas were measured during a movement of the dominant right hand in 15 patients with Parkinson's disease deprived of their usual levodopa treatment, in 11 patients with Parkinson's disease undergoing long-term treatment with levodopa, and in 15 normal volunteers. The supplementary motor areas were significantly activated in the normal subjects and in the patients receiving levodopa but not in the patients deprived of levodopa. The contralateral primary sensory motor area was significantly activated in all three groups. The ipsilateral primary sensory motor cortex was not activated in the normal subjects and the non-treated patients but was in the patients treated with levodopa. It is concluded that the supplementary motor area hypoactivation which is observed in akinetic nontreated patients with Parkinson's disease is not present in patients undergoing long-term treatment with levodopa. This result suggests that (a) levodopa improves the functional activity of supplementary motor areas in Parkinson's disease and (b) there is no pharmacological tolerance to this effect. The ipsilateral primary motor cortex activation observed in the patients treated with levodopa could be related to levodopainduced abnormal involuntary movements.
\end{abstract}

$(\mathcal{F}$ Neurol Neurosurg Psychiatry 1994;57:567-571)

Brain mapping of active areas playing a part in voluntary movements by measuring regional cerebral blood flow (rCBF) has suggested that

Table 1 Clinical features of subjects studied

\begin{tabular}{lllc}
\hline & & \multicolumn{2}{l}{ Patients with Parkinson's disease } \\
\cline { 3 - 4 } Feature & $\begin{array}{l}\text { Normal subjects } \\
(n=15)\end{array}$ & $\begin{array}{l}\text { Non-treated } \\
(n=15)\end{array}$ & $\begin{array}{c}\text { Levodopa } \\
(n=11)\end{array}$ \\
\hline Age (years) & $54(11)$ & $60(8)$ & $63(6)$ \\
Duration of Parkinson's disease (years) & - & $6(4)$ & $7(6)$ \\
Duration of levodopa treatment (years) & - & $4(5)$ & $6(4)$ \\
Levodopa dose (mg/day) & - & $466(333)$ & $580(417)$ \\
Hoehn and Yahr" "off" & - & $2 \cdot 3(1 \cdot 0)$ & $2 \cdot 4(1 \cdot 2)$ \\
UPDRS “off" & - & $23(10)$ & $25(7)$ \\
UPDRS "on" & - & $7(9)$ & $9(7)$ \\
\hline
\end{tabular}

No significant difference was observed for any parameter. Age mean (SD) values were compared using an analysis of variance. Non-treated and levodopa Parkinson's disease groups were compared using a $t$ test or a Mann-Whitney $U$ test when necessary. ${ }^{\star}$ UPDRS = Unified Parkinson's Disease Rating Scale. ${ }^{18}$ the activity of the supplementary motor area (SMA) is defective in akinetic patients with Parkinson's disease. ${ }^{1-3}$ This result agrees with anatomical, ${ }^{4-5}$ clinical, $^{6-7}$ and electrophysiological $^{8}$ studies which support a role for the SMA in the pathophysiology of akinesia in Parkinson's disease (for review, see Marsden'). It has been shown that apomorphine reverses the impaired activation of $\mathrm{rCBF}$ in the SMA in Parkinson's disease. ${ }^{2-3}$ To our knowledge, the effects of levodopa have not yet been investigated. Moreover, when the $\mathrm{rCBF}$ is measured in the resting state, the effects of levodopa and apomorphine disappear within a few days of treatment and tolerance occurs. ${ }^{1011}$ This study was conducted to assess whether the motor activation in the SMA is normal or impaired when patients with Parkinson's disease are treated long term with levodopa.

\section{Subjects and methods}

SUBJECTS

Twenty-six right-handed patients with Parkinson's disease entered the study (17 men, nine women). All were clinically diagnosed as having "idiopathic" Parkinson's disease. ${ }^{12}$ All had a clear positive response to long-term dopaminergic treatment and acute challenges of levodopa ${ }^{13}$ or apomorphine. ${ }^{14}$ None was demented. Patients with clinical features suggestive of progressive supranuclear palsy ${ }^{15}$ or multiple system atrophy ${ }^{16}$ were excluded from the study. All patients were chosen to have an akinetic-rigid syndrome without tremor to avoid motor cerebral activation related to this involuntary movement. ${ }^{17}$ All patients had bilateral or right-side predominant symptoms. The 26 patients with Parkinson's disease were divided in two groups. Both groups were carefully defined to avoid relevant demographic, clinical, or therapeutic differences (table 1 ).

The first group included 15 patients with Parkinson's disease who were studied in the "off" condition-that is, after levodopa and other drugs for Parkinson's disease (bromocriptine in five patients, selegiline in four patients) had been withheld for a delay long enough (at least 24 hours) to allow the reappearance of the parkinsonian symptoms. These patients were clinically evaluated at the time of the rCBF measurements in the "off" condition with the global motor examination score of the Unified Parkinson's Disease Rating Scale (UPDRS) ${ }^{18}$ and the Hoehn and Yahr scale. ${ }^{19}$ Patients in this group were also 
evaluated (UPDRS motor examination) during the week preceding the rCBF study while normally treated with levodopa and their usual drugs (table 1). This procedure allowed us to evaluate the non-treated patients in the "on" condition to check that levodopa induced a comparable motor improvement in this and the other group. Six patients of this group presented mild peak dose dyskinesia (scored less than 2 for the items duration and disability of dyskinesia of the UPDRS subscale complications of treatment) when receiving their usual treatment. Six patients in this group have already been reported elsewhere. ${ }^{2}$

The second group included 11 other patients with Parkinson's disease who were studied while levodopa and other usual longterm treatments for Parkinson's disease (bromocriptine in five patients, selegiline in three patients) were not interrupted. Patients were examined four to six weeks before the rCBF study in the "off" condition (UPDRS motor examination and Hoehn and Yahr score after at least 24 hours of no drug treatment) to check that the motor impairement induced by the disease was similar in the nontreated and levodopa groups. Patients were also evaluated (motor examination of the UPDRS) in the "on" condition at the time of the rCBF measurements (table 1). The rCBF measurements were performed during "on" periods in the levodopa patients who had motor fluctuations. Levodopa patients had no visible (five patients) or mild to moderate (six patients) abnormal involuntary levodopainduced movements in the "on" condition (scored 2 or less for the items duration and disability of dyskinesia on the UPDRS subscale complications of treatment).

The two groups of patients with Parkinson's disease were compared with 15 normal volunteers (15 men, mean (SD) age 54 (11) years). None of these normal subjects had any history of neurological disease and none had received any treatment.

Informed consent was obtained from all patients and normal subjects. The project was approved by the local ethics committee.

\section{PARADIGM DESIGN}

Two rCBF measurements were obtained the same day in each subject with a 60 minute interval between each scan: the first measurement was obtained during the execution of a motor task-that is, during movement of the right hand-and the second in the resting state. Motor task and resting states were balanced for visual and auditory stimuli and were performed under conditions of sensory deprivation.

The motor task has been described previously. ${ }^{320}$ It consisted of sequential finger to thumb opposition movements in turn lasting for the four minutes of the rCBF data acquisition period. We measured the way each subject performed the motor task using video recordings of the hand movements. This procedure allowed us to measure the motor task's frequency (number of fingers to thumb oppositions each minute) and amplitude (0 to 3 scale). All subjects were pretrained to execute the motor task until they had shown that they were able to perform the movement with a stable amplitude and frequency. Special efforts were made to arrange the experiment so that the three groups executed the motor task in a similar quantitative way. For this purpose, the non-treated patients with Parkinson's disease (that is, those who had the greatest difficulties in executing the motor task because of akinesia) were studied first. They were told to execute the motor task "as well as they could with the largest possible amplitude and the most regular moderate frequency". The rCBF measurements were performed when the patients had found their best and stable performance. The mean frequency and mean amplitude of the non-treated patients were then calculated using the video recordings (frequency $45 /$ minute, amplitude 1.5). The other subjects (levodopa patients and normal controls) were studied afterwards. They had the capacity to execute the motor task more rapidly and with a larger amplitude than the non-treated patients, but they were told to fit their own performance to that of the non-treated patients. The rCBF measurements were performed when they had shown that they could execute the motor task with the appropriate required performance. At the end of the study, the video recordings of all the subjects were analysed blindly to compare the mean amplitude and frequency of the three groups. In every patient the absence or presence of contralateral involuntary movements during the execution of the motor task was assessed visually and on the video recording.

\section{RCBF MEASUREMENT}

The technique used to measure $\mathrm{rCBF}$ has been described previously. ${ }^{2}$ Briefly, $\mathrm{rCBF}$ was assessed using SPECT (Tomomatic 64, Medimatic, Copenhagen, Denmark) and intravenous injection of ${ }^{133} \mathrm{Xe}(2220 \mathrm{MBq}){ }^{21}$ The mean global flow was measured from data collected from three transverse slices simultaneously at 0,4 , and $8 \mathrm{~cm}$ above the canthomeatal plane. rCBF changes were studied in three regions of interest (ROIs) from slice 3: a medial anterior ROI corresponding to the two SMAs (12 pixels) and two symmetrical and lateral ROIs (16 pixels each) corresponding to the left (contralateral to the movement) and right (ipsilateral to the movement) primary sensory motor areas (contro S1M1 and ipsi S1M1 respectively) (figure). Subcortical motor ROIs from slices 2 and 3 were not analysed because we previously could not detect any significant changes with this technique in these regions. ${ }^{2}$ The $\mathrm{rCBF}$ data obtained in the three cortical motor ROIs were expressed after normalisation with a global factor calculated from the global CBF of the three slices ( $\mathrm{rCBF}$ at rest/rCBF during motor task). The rCBF value of each ROI collected during the motor task was multiplied by this factor to eliminate non-specific rCBF changes. Localisation of the ROIs on the SPECT scans were carried out blind. With respect to the spatial resolution of our tomog- 
Table 2 Mean (SD) rate (number each minute) and amplitude (0-3 scale) of the motor task

\begin{tabular}{llll}
\hline & \multicolumn{2}{l}{ Patients with Parkinson's disease } \\
\cline { 3 - 4 } & $\begin{array}{l}\text { Normal subjects } \\
(n=15)\end{array}$ & $\begin{array}{l}\text { Non-treated } \\
(n=15)\end{array}$ & $\begin{array}{c}\text { Levodopa } \\
(n=11)\end{array}$ \\
\hline Rate & $40(17)$ & $45(20)$ & $39(17)$ \\
Amplitude & $1.7(0.4)$ & $1.5(0.7)$ & $2.0(0 \cdot 7)$ \\
\hline
\end{tabular}

There was no statistical difference between the rate and amplitude among the three groups (multivariate analysis of variance).

Table $3 \mathrm{rCBF}$ values ( $\mathrm{ml} / \mathrm{min} / 100 \mathrm{~g}$ ) at rest and during motor activation in the three cortical motor regions of interest

\begin{tabular}{|c|c|c|c|c|c|c|}
\hline & & & Patients & ith Parkinson & disease & \\
\hline & $\begin{array}{l}\text { Normal } \\
(n=15)\end{array}$ & ubjects & $\begin{array}{l}\text { Non-trea } \\
(n=15)\end{array}$ & & $\begin{array}{l}\text { Levodopo } \\
(n=11)\end{array}$ & \\
\hline & Rest & Motor task & $\overline{R e s t}$ & Motor task & Rest & Motor task \\
\hline $\begin{array}{l}\text { Contralateral S1M1 } \\
\text { SMA } \\
\text { Ipsilateral S1M1 }\end{array}$ & $\begin{array}{l}56(9) \\
61(10) \\
57(11)\end{array}$ & $\begin{array}{l}61(10)^{\star \star} \\
64(12)^{\star} \\
56(10)^{4}\end{array}$ & $\begin{array}{l}59(10) \\
65(14) \\
60(10)\end{array}$ & $\begin{array}{l}61(9)^{\star} \\
63(12) \\
59(10)\end{array}$ & $\begin{array}{l}53(7) \\
56(10) \\
52(8)\end{array}$ & $\begin{array}{l}57(8)^{\star \star} \\
61(10)^{\star \star} \\
56(10)^{\star}\end{array}$ \\
\hline
\end{tabular}

rCBF significantly increased during the motor activation in the contralateral S1M1 of the three groups, in the SMA of the normal subjects and the levodopa treated patients but not of the nontreated patients, and in the ipsilateral S1M1 of the levodopa treated patients but not of the two other groups (paired Student $t$ test: ${ }^{\star} \mathrm{p}<0.05 ;{ }^{\star \star} \mathrm{p}<0.01$ ).

raphy system, we must admit that the rCBF values in such ROIs may represent the partial value of the specified anatomical regions. The $\mathrm{PaCO}_{2}$ was continuously recorded using a cutaneous electrode and a $\mathrm{PaCO}_{2}$ monitor (Kontron 634, Kontron, Basle, Switzerland). A small blood sample was withdrawn for determination of the packed cell volume. Systolic and diastolic blood pressure were measured at the moment of each rCBF measurement.

\section{STATISTICAL ANALYSIS}

The rCBF values were compared between the resting state and the motor task state in the three ROIs of each group using a paired Student's $t$ test. A comparison of the percentages of $\mathrm{rCBF}$ changes $(\mathrm{rCBF} \% \Delta)$ induced by the execution of the motor task in the three ROIs between the three groups of subjects were performed using a multivariate analysis of variance (MANOVA). A post hoc univariate analysis (Duncan test) was examined fol-

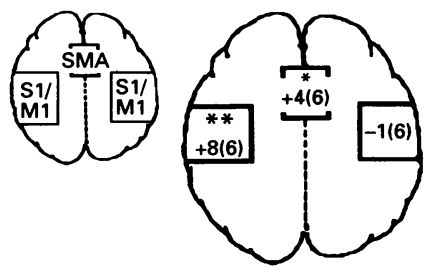

Normals

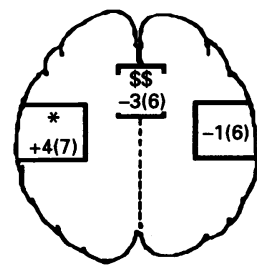

Non-treated

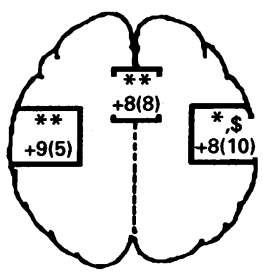

Levodopa treated
Mean percentages of changes in $r C B F(r C B F \% \Delta)$ induced by motor activation in the three cortical motor regions of interest (ROIs). The small scheme in the top left-hand corner of the figure shows the position of the three ROIs studied on the third slice of the tomograph. Intragroup $r C B F$ changes between activation and rest are compared in each $R O I$ by the paired Student's $t$ test $\left({ }^{\star} p<0.05, \star \star p<0.01\right)$. Intergroup $r C B F \% \Delta$ values are compared in each ROI using a multiple analysis of variance (significant effect of the interaction "group" by "ROIs", $p<0.05)$. The contralateral S1M1 $r C B F \% \triangle$ was not significantly different among the three groups. Conversely, the SMA rCBF\% $\%$ was significantly smaller in the "non-treated" group than in the other groups ( $\$ \$=p<0.01$ ) and the ipsilateral $S 1 M 1 r C B F \% \triangle$ was significantly larger in the "levodopa treated" group than in the two other groups $(\$=p<0.05)$. lowing a significant $F$ test. Significance was accepted for $p<0.05$. Results are expressed as mean (SD).

\section{Results}

There was no significant difference in the frequency and amplitude of the motor task between the three groups (MANOVA; table 2). One normal subject, two non-treated patients, and one levodopa patient isotonically contracted the muscles of the left arm or leg, or both (with no displacement) when they executed the motor task. During the motor activation scan, one levodopa patient had a mild but obvious tremor of the left hand (less than $10 \mathrm{~mm}$ amplitude, $4 \mathrm{~Hz}$ frequency) which had not previously been noticed when the patient was selected for the study. Five other levodopa patients presented some abnormal involuntary movements during the execution of the motor task: one had a dystonic contraction of the right foot for $1.5 \mathrm{~min}$ utes and the four others had mild dyskinetic movements of the face, left hand or leg.

There was no significant difference between the mean global CBF of the three groups at rest and during the execution of the motor task. There was no difference between blood pressure, $\mathrm{PaCO}_{2}$ and packed cell volume between the three groups and in the same group from one scan to the other. At rest, in each group there was no difference in rCBF between the ipsilateral and contralateral S1M1. There was no difference between the different corresponding ROIs of the three groups at rest.

The execution of the motor task induced a significant increase in $\mathrm{rCBF}$ in the contralateral S1M1 $(p<0.01)$ and in the SMA $(p<0.05)$ of the normal subjects. The same movement only induced a significant increase in $\mathrm{rCBF}$ in the contralateral S1M1 $(\mathrm{p}<0.05)$ but not in the SMA of the non-treated patients. Finally, the motor activation induced a significant increase in rCBF in the contralateral S1M1 $(p<0.01)$, in the ipsilateral S1M1 $(\mathrm{p}<0.05)$, and in the SMA $(\mathrm{p}<0.01)$ of the levodopa patients (table 3 ).

When we compared the $\mathrm{rCBF} \% \Delta$ in the three different ROIs of the three different groups, the MANOVA showed a significant effect of the interaction group by ROIs $(p<0.05)$. A post hoc univariate analysis (Duncan test) showed that there was no significant difference between the three groups in the contralateral $S 1 M 1 \mathrm{rCBF} \% \Delta$. On the contrary, the SMA rCBF\% $\Delta$ was significantly smaller in the non-treated group than in the normal and the levodopa groups $(p<0.01)$. Finally, the ipsilateral S1M1 rCBF\% $\Delta$ was significantly larger in the levodopa group than in the normal $(p<0.05)$ and the "nontreated" ( $p<0.01)$ groups (figure).

\section{COMMENTS}

These results show that the SMA was not normally activated in akinetic patients with Parkinson's disease deprived of levodopa, though this abnormality was not observed in patients with Parkinson's disease treated long 
term with levodopa. An ipsilateral primary motor cortex hyperactivation was also detected in these latter patients.

We studied two different groups of patients with Parkinson's disease. Consequently, we do not have data on the same subjects studied prospectively without and with levodopa as in our previous study with apomorphine. ${ }^{2}$ This different procedure was chosen because we wanted to study the effects of a long-term treatment rather than the effects of an acute challenge of levodopa. Our ethics committee stated that it was not recommended to perform four repeated scans within a short time in the same patient because of the total radioactive dose. Therefore, we had to compare rCBF changes in two different groups of patients. For this reason we carefully matched the demographic, clinical, and therapeutic features of the patients to reduce the risk that differences in rCBF activation could be due to factors other than levodopa (table 1).

We also controlled the way the motor task was executed by the three groups of subjects. When a motor task is executed with different frequencies, the number of task iterations within the measurement interval (four minutes in this instance) varies. This can lead to differences in mean neuronal activity and in turn to different changes in the locally measured rCBF. This phenomenon has already been reported in the primary visual cortex..$^{22}$ We showed the same phenomenon in the contralateral S1M1 of the normal volunteers. ${ }^{24}$ The three different groups of subjects in this study executed the motor task with the same mean amplitude and the same mean frequency (table 2). This allows us to exclude the possibility that intergroup differences in $\mathrm{rCBF}$ activation could be due to differences in task execution. It could be suggested that artificially restraining the rate and amplitude of finger oppositions could artefactually increase SMA activation in the control and the levodopa groups by additionally requiring monitoring of movement. We have previously shown under comparable conditions, however, that the rate and amplitude variations of the motor task only mildly and non-significantly influence SMA activation in normal volunteers. ${ }^{24}$

The activation of the SMA was impaired in the non-treated patients with Parkinson's disease. This has already been reported in other studies and emphasises the hypothesis that a functional deafferentation of the SMA plays a part in the pathophysiology of parkinsonian akinesia. ${ }^{1-3}$ This deafferentation is probably related to a defective outflow from the basal ganglia to the cortical motor areas. ${ }^{25} 26$ Our results also show, for the first time to our knowledge, that the SMA is normally activated in the levodopa patients with Parkinson's disease.

These data suggest that levodopa is able to improve the cortical SMA hypoactivation in Parkinson's disease in a comparable way to the direct dopamine agonist apomorphine. ${ }^{23}$ Moreover they suggest that no tolerance to this phenomenon occurs because patients were treated long term. This is in contrast with the short-lived effects of levodopa and apomorphine on $\mathrm{rCBF}$ in the resting state. ${ }^{1011}$ It is therefore possible to describe two different effects of dopamine drugs on rCBF in patients with Parkinson's disease. The first has been observed in the resting state. ${ }^{1011} \mathrm{It}$ corresponds to a global direct vasodilation of cerebral blood vessels and in this instance tolerance occurs. The second is observed during motor activation. ${ }^{23}$ In this last instance there is no tolerance and it is reasonable to suggest that the $\mathrm{rCBF}$ increase in the SMA reflects an improvement in the underlying neuronal activity. This improvement is probably secondary to an increase in pallido-thalamo-cortical outflow related to the dopaminergic effects of the drug in the basal ganglia. ${ }^{26}$

In this study the contralateral S1M1 was significantly activated in the three groups. The movement was executed similarly by the three groups and the activation of this area was not significantly different among the groups. This result suggests two remarks. Firstly, the activity of the primary motor cortex is not markedly impaired in patients with Parkinson's disease and the main pathophysiological mechanisms of akinesia probably affect other parts of the motor system. ${ }^{1}$ Secondly, the activation of the primary motor cortex is more closely linked to the execution of the movement than that of the SMA and the two areas probably have different motor functions. ${ }^{24}$ It can be seen, however, that rCBF activation of the contralateral S1M1 was half the size in the non-treated group than in the two other groups $(+4 \% v+8 \%)$. The difference among groups was not significant but could suggest that motor activation was not strictly normal in the primary motor cortex of patients with Parkinson's disease in the "off" condition. This observation is perhaps related to a direct influence of the basal ganglia on the primary motor cortex..$^{25}$

The significant increase in $\mathrm{rCBF}$ in the ipsilateral S1M1 of the levodopa treated patients was unexpected and is more difficult to elucidate. The possibility that a technical artefact explains this result remains. We have, however, studied several dozen normal subjects and patients with this technique. ${ }^{24}$ We have never observed such a pattern of rCBF motor activation except in the levodopa group. Our rCBF data are normalised by applying a correction factor for global rCBF changes. This procedure markedly reduces the risk of considering non-specific rCBF signals. A bilateral increase in rCBF has been observed with positron emission tomography in the parietal cortex of normal subjects performing a motor task. ${ }^{27}$ We admit that rCBF in the ROIs defined here may represent only a partial value of the specified anatomical regions because of the limits of the spatial resolution of our tomograph. The parietal cortex is thus perhaps involved in some aspects of the bilateral motor activation observed here. If this explanation were true, however, we cannot understand why such a signal was so clearly detected in the levodopa group only and not in the normal subjects and the non-treated group. 
We therefore believe that the ipsilateral S1M1 activation of the levodopa group was specific and suggest that the ipsilateral S1M1 affected in some way the motor behaviour of our patients treated long term with levodopa. In fact, a bilateral activation of the primary motor cortex has also been observed by others in patients who had recovered motor function after ischaemic stroke ${ }^{20} 28$ and in patients with lateral amyotrophic sclerosis (D. Brooks, personal communication).

The hypothesis that the ipsilateral S1M1 could directly affect the motor circuit underlying the movement in Parkinson's disease is unlikely because there was no ipsilateral S1M1 activation in the non-treated patients. Conversely, it is well known from clinical observation that when a patient with Parkinson's disease concentrates to execute a motor or intellectual task, the occurence or reappearance of involuntary movements is facilitated.

We observed that several subjects in this study (one normal, two non-treated patients, and one levodopa patient) showed an involuntary isotonic contraction of the opposite side when performing the motor task. It is, however, hard to believe that such a small contraction could induce such a large $(+8 \%)$ increase in rCBF because the S1M1 activity is dependent on the rate and amplitude of the movement. ${ }^{24}$ This hypothesis does not explain why the ipsilateral S1M1 would not have been significantly activated in the normal subject and in the non-treated patients who also showed some contralateral isotonic contraction.

A mild rest tremor of the left hand was observed in one levodopa patient during motor activation. Tremor increases the rCBF in $S 1 M 1$ and this could explain a bilateral S1M1 activation in this patient. ${ }^{17}$

Five other levodopa patients presented moderate visible levodopa-induced dystonic or dyskinetic movements during the execution of the motor task. The abnormal involuntary movements affected the left limbs in three patients and were observed on both sides in the other two. It has been suggested that the pathophysiology of hyperkinetic diseases could be the "opposite" of that of hypokinetic disorders. ${ }^{26} \mathrm{We}$ can thus imagine that levodopa-induced dyskinesia could be due to an overactivity of the subcorticocortical pathways originating from the thalamus and projecting to the cortical motor areas. It is interesting to note in our levodopa patients that the hyperactivation of the ipsilateral S1M1 was not isolated. In fact, the magnitude of the SMA activation was twice as large (though this was not statistically significant) in these patients than in the normal subjects $(8 v 4)$. It is thus tempting to speculate that the cortical motor hyperactivation observed in the ipsilateral S1M1 and in the SMA of levodopa-treated patients was related to an excess of subcorticocortical outflow possibly related to some dopaminergic "supersensitivity" as suggested in the pathophysiology of levodopa-induced dyskinesia. We are now conducting further studies to compare the motor activation in patients with Parkinson's disease with and without levodopa-induced dyskinesia to test this hypothesis.

We thank Therese Pujol, Chantal Blanchard, and Gerard Viallard for technical assistance, and the nurses of the Movement Disorders and Clinical Neuropharmacology Unit for taking care of the patients.

1 Playford E, Jenkins I, Passingham R, Nutt J, Frackowiak R, Brooks D. Impaired mesial frontal and putamen activation in Parkinson's disease: a positron emission tomography study. Ann Neurol 1992;32:151-61.

2 Rascol O, Sabatini U, Chollet F, et al. Supplementary and primary sensory motor area activity in Parkinson's disease. Regional cerebral blood flow changes during fingers movements and effects of apomorphine. Arch Neurol 1992;49:144-8.

3 Jenkins I, Fernandez W, Playford E, et al. Impaired activation of the supplementary motor area (SMA) in Parkinson's disease is reversed when akinesia is treated with apomorphine. Ann Neurol 1992;32:749-57.

4 Jurgens U. The efferent and afferent connections of the supplementary motor area. Brain Res 1984;300:63-87.

5 Schell G, Strick P. The origin of the thalamic inputs to the arcuate premotor and supplementary motor areas. f Neurosci 1984;4:539-60.

6 Dick J, Benecke R, Rothwell J, Day B, Marsden C. Simple and complex movement in a patient with infarction of the right supplementary motor area. Mov Disord 1986; 1:255-66.

7 Obeso J, Artieda J, Oliver A, Muruzabal J, BarraquerBordas $L$. Motor performance following the bilateral lesion of the supplementary motor area (SMA) in man. f Physiol 1991;438:31.

8 Dick J, Rothwell J, Day B, et al. The Bereitschaftspotential is abnormal in Parkinson's disease. Brain 1989;112: 233-44.

9 Marsden C. Slowness of movement in Parkinson's disease. Mov Disord 1989;4(suppl):26-37.

10 Leenders K, Wolfson L, Gibbs J, et al. The effects of Ldopa on regional cerebral blood flow and oxygen metabolism in patients with Parkinson's disease. Brain olism in patients

11 Sabatini U, Rascol O, Celsis $P$, et al. Subcutaneous apomorphine increases regional cerebral blood flow in parkinsonian patients via peripheral mechanisms. $\mathrm{Br} \mathcal{F}$ Clin Pharmacol 1991;32:229-34.

12 Gibb W, Lees A. The relevance of Lewy body to the pathgenesis of idiopathic Parkinson's disease. F Neurol Neurosurg Psychiatry 1988;51:745-52.

13 Esteguy M, Bonnet AM, Kefalos J, Lhermitte F, Agid Y. Le test a la L-dopa dans la maladie de Parkinson. Rev Neurol 1985;141:413-5.

14 Hughes A, Lees A, Stern G. Apomorphine test to predict dopaminergic responsiveness in parkinsonian syndromes. Lancet 1990;ii:32-4.

15 Lees A. The Steele-Richardson-Olszewski syndrome (progressive supranuclear palsy). In: Marsden C, Fahn S,
eds. Movement Disorders. Vol. 2. London: Butterworth, eds. Movement

16 Quinn N. Multiple system atrophy-the nature of the beast. F Neurol Neurosurg Psychiatry 1989;suppl:78-89.

17 Colebatch J, Findley L, Frackowiak R, Marsden C, Brooks $D$. Preliminary report: activation of the cerebellum in essential tremor. Lancet 1990;2:1028-30.

18 Fahn $S$, Elton $R$ and the members of the Unified Parkinson's Disease Rating Scale Development Committee. Unified Parkinson's Disease Rating Scale. In: Fahn S, Marsden C, Calne D, eds. Recent developments in Parkinson's disease. New York: MacMillan, 1987:153-63.

19 Hoehn M, Yahr M. Parkinsonism: onset, progression and mortality. Neurology 1967;17:427-42.

20 Chollet F, Di Piero V, Wise R, Brooks D, Dolan R, Frackowiak $R$. The functional anatomy of motor recovery after stroke in humans: a study with positron emission after stroke in humans: a study with posit
tomography. Ann Neurol 1991;29:63-71.

21 Celsis P, Goldman T, Henriksen L, Lassen N. A method for calculating regional cerebral blood flow from emisfor calculating regional cerebral blood flow from emission computed tomography of inert gas
$f$ Comput Assist Tomogr 1981;5:641-5.

22 Fox $P$, Raichle $M$. Stimulus rate dependence of regional cerebral blood flow in human striate cortex demonstrated by positron emission tomography. $\mathcal{F}$ Neurophysiol 1984;51:1109-20.

23 Fox $P$, Raichle $M$. Stimulus rate determines regional brain blood flow in striate cortex. Ann Neurol 1985;17:303-5.

24 Sabatini U, Chollet F, Rascol O, et al. Effect of the side and rate of stimulation on cerebral blood flow changes in motor areas during finger movements in humans. 7 Cereb Blood Flow Metab 1993;13:639-45.

25 Alexander G, Crutcher M. Functional architecture of basal ganglia circuits: neural substrates of parallel probasal ganglia circuits: neural substrates of
cessing. Trends Neurosci 1990;13:266-71.

26 Delong $M$. Primate models of movement disorders of basal ganglia origin. Trends Neurosci 1990;13:381-5.

27 Deiber MP, Passingham R, Colebatch J, Friston K, Nixon Peiber MP, Passingham R, Colebatch J, Friston K, Nixon movements: a study with positron emission tomography. movements: a study with positron

28 Weiller C, Chollet F, Friston K, Wise R, Frackowiak R. Functional reorganization of the brain in recovery from striatocapsular infarction in man. Ann Neurol 1992; 31:463-72. 